\title{
Self-similarly expanding networks to curve shortening flow
}

\author{
Oliver C. SCHNÜRER AND FEliX SCHUlze
}

\begin{abstract}
We consider a network in the Euclidean plane that consists of three distinct half-lines with common start points. From that network as initial condition, there exists a network that consists of three curves that all start at one point, where they form 120 degree angles, and expands homothetically under curve shortening flow. We also prove uniqueness of these networks.
\end{abstract}

Mathematics Subject Classification (2000): 53C44 (primary); 35Q51, 74K30, 74N20 (secondary).

\section{Introduction}

We consider networks in $\mathbb{R}^{2}$ consisting of three curves with common start points that evolve under curve shortening flow. As curve shortening flow is the $L^{2}$-gradient flow for the length functional, it is natural to assume that each pair of curves encloses an angle of $120^{\circ}$ at their common start points. If such is the case, we say that the curves fulfill the balancing condition. In this paper, we investigate curve shortening flow of networks for which the balancing condition may be violated initially. More precisely, we study three half-lines with common start points. We prove that there exists a self-similarly expanding network that approaches the union of the three half-lines for small times, evolves under curve shortening flow, and fulfills the balancing condition for positive times. Moreover, we show that such a solution is unique.

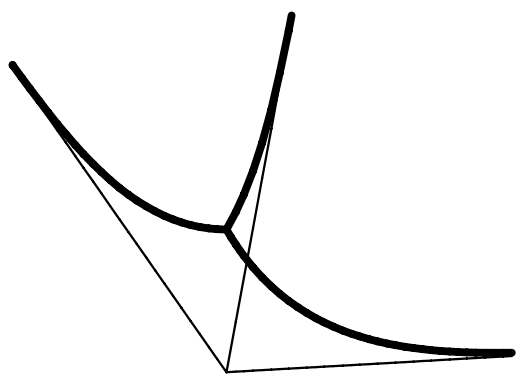

In order to formulate our existence and uniqueness result more precisely, let $l_{1}, l_{2}$,

The authors were supported by SFB 647/B3 "Raum - Zeit - Materie": Singularity structure, long-time behavior and dynamics of non-linear evolution equations.

Received March 13, 2007; accepted in revised form October 18, 2007. 
and $l_{3}$ be three distinct half-lines that start in the same point which we assume to be the origin in $\mathbb{R}^{2}$. We want to find homothetically expanding solutions to curve shortening flow for an initial configuration as described above. Such solutions form a family of networks $M_{t}, t \geq 0$. Each network consists of three curves $\alpha_{i}:(\cdot, t)$ : $[0, \infty) \rightarrow \mathbb{R}^{2}$ such that

- $\alpha_{i}(r, t), i=1,2,3$, is smooth for $t>0$ and continuous up to $t=0$,

- for $t>0$, the curves $\alpha_{i}(\cdot, t), i=1,2,3$, are regular, i.e.

$$
\frac{\partial}{\partial r} \alpha_{i}(r, t) \neq 0
$$

uniformly up to $r=0$,

- the start points $\alpha_{i}(0, t), i=1,2,3$, coincide for all times $t>0$, but may depend on time,

- $\alpha_{i}(\cdot, t), i=1,2,3$, are three embedded curves and for all $t$ the three curves only meet at the start point,

- the tangent vectors at the start point fulfill the balancing or $120^{\circ}$ condition, i.e.

$$
\sum_{i=1}^{3} T_{i}=0 \text { for all } t>0
$$

where

$$
T_{i}=\frac{\left.\frac{\partial}{\partial r} \alpha_{i}(r, t)\right|_{r=0}}{\left|\frac{\partial}{\partial r} \alpha_{i}(r, t)\right|_{r=0} \mid}
$$

denote the tangent vectors to the curves at $(0, t)$,

- each curve connects to infinity, i.e.

$$
\lim _{r \rightarrow \infty}\left|\alpha_{i}(r, t)\right|=\infty \text { for all } t \geq 0 \text { and } i=1,2,3,
$$

- the curve $\alpha_{i}(\cdot, t), i=1,2,3, t \geq 0$, is at infinity asymptotically close to the half-line $l_{i}$, i.e. denoting by $d_{\mathcal{H}}$ the Hausdorff distance,

$$
d_{\mathcal{H}}\left(\alpha_{i}([0, \infty), t) \cap\left(\mathbb{R}^{n} \backslash B_{r}(0)\right), l_{i} \cap\left(\mathbb{R}^{n} \backslash B_{r}(0)\right)\right) \rightarrow 0 \text { for } r \rightarrow \infty,
$$

- each curve flows for $r>0$ according to curve shortening flow (which equals mean curvature or Gauß curvature flow in one space dimension; more precisely, in general, we have to add a non-zero tangential velocity in order to avoid problems with the parameterization - we will later rewrite this flow equation equivalently as a graphical flow equation and thereby fix a parameterization), i.e.

$$
\left(\frac{d}{d t} \alpha_{i}\right)^{\perp}=\Delta \alpha_{i},
$$

where $\Delta$ denotes the Laplace-Beltrami operator on the curve, 
- for $t=0$, we get our initial configuration,

$$
\left\{\alpha_{i}(r, 0): r \geq 0\right\}=l_{i} \text { for } i=1,2,3 \text {, }
$$

- the solution is homothetically expanding, i.e. for $0<t_{1}<t_{2}$, there exists $\lambda>1$ such that

$$
\lambda \cdot \bigcup_{i=1}^{3}\left\{\alpha_{i}\left(r, t_{1}\right): r \geq 0\right\}=\bigcup_{i=1}^{3}\left\{\alpha_{i}\left(r, t_{2}\right): r \geq 0\right\},
$$

- $\alpha_{i}$ is of class $C^{0}([0, \infty) \times[0, \infty)) \cap C^{\infty}([0, \infty) \times(0, \infty))$ for $i=1,2,3$.

Definition 1.1. A family of curves $\left(\alpha_{i}\right)_{i=1,2,3}$ as described above is said to be a network which expands homothetically under curve shortening flow from three half-lines. In particular, we impose the balancing condition for all positive times. We say that two such networks are equal if their images $\bigcup_{i=1}^{3} \alpha_{i}([0, \infty), t)$ in $\mathbb{R}^{2}$ coincide.

In the situation described above, we prove the following

Theorem 1.2. For an initial configuration consisting of three distinct half-lines starting at the origin, there exists a unique family of networks, expanding homothetically under curve shortening flow that converges to the three given half-lines for $t \searrow 0$ in Hausdorff distance and in $C^{\infty}$-topology away from the triple point.

In this paper we use a definition of network which requires the network to be "treelike", i.e. it contains no loops. This is perfectly appropriate in our situation as there are no homothetically expanding networks with a more complicated topology asymptotic to three half-lines at infinity. This is shown in Remark A.1.

The strategy of the proof is as follows. For self-similar solutions to curve shortening flow, we can reduce the parabolic equation to an elliptic equation which describes self-similar solutions at a fixed positive time, e.g. at $t=1 / 2$. Then we solve this elliptic equation with prescribed start point $P=\alpha_{i}(0,1 / 2)$ such that $\alpha_{i}(\cdot, 1 / 2)$ connects to infinity and is asymptotically close to the half-line $l_{i}$. This means that $M_{t}=\alpha_{i}([0, \infty), t)$ is a family of curves that expand homothetically, is asymptotic to $l_{i}$ at spatial infinity and $\alpha_{i}(0,1 / 2)=P$. It is not to be expected that prospective solutions will fulfill the balancing condition at $P$ for arbitrary $P \in \mathbb{R}^{2}$. In general, these curves $\alpha_{i}(\cdot, t)$ fulfill all the properties required above for a selfsimilarly expanding solution but not necessarily the balancing condition

$$
\sum_{i=1}^{3} T_{i}=0
$$

We will show, however, that for fixed distinct half-lines $l_{i}$, the scalar product

$$
\left\langle P, \sum_{i=1}^{3} T_{i}\right\rangle
$$


is negative for large values of $|P|$. As the tangent vectors $T_{i}$ depend continuously on $P$, a mapping degree argument yields that there exists some $P_{0} \in \mathbb{R}^{2}$ such that

$$
\sum_{i=1}^{3} T_{i}=0,
$$

i.e. by choosing $P_{0}$ as described, we can also ensure that the balancing condition is fulfilled. So we obtain a homothetically expanding network.

Uniqueness follows from the observation that for a self-similarly expanding solution that intersects a family of self-similarly expanding solutions (all solutions are self-similarly expanding under the same homotheties) that are all asymptotic to a fixed half-line at infinity, the angle, at which these curves intersect, is monotone along the solution mentioned at the beginning. This follows from applying the Gauß-Bonnet theorem and the divergence theorem and uses also the fact that we consider self-similarly expanding solutions to curve shortening flow.

The convergence of a smooth, closed and embedded curve in $\mathbb{R}^{2}$ to a 'round' point was shown by M. Gage and R. Hamilton [5] and M. Grayson [6]. An alternative proof was given by G. Huisken in [7]. Short-time existence for curve shortening flow of an embedded network of three curves meeting at one common point, at which the balancing condition is satisfied, was shown by C. Mantegazza, M. Novaga, and V. Tortorelli [9]. Provided that no type II singularities occur and under some further natural assumptions, they show that such a network converges to a minimal configuration as $t \rightarrow \infty$. The problem of the existence of a smooth network, evolving by curve shortening flow and satisfying the balancing condition with an initial network not satisfying the balancing condition is raised in that paper. In the present paper we give a partial answer to this problem.

The evolution of embedded networks, satisfying the balancing condition, arises naturally in two-dimensional multi-phase systems, where the evolution of the interfaces between different phases can be modeled by curve shortening flow. This comes from the fact that in certain models the total energy of the interface is proportional to the total length of the interface, and curve shortening flow is the $L^{2}$ gradient flow of the length functional. An example for such a model is given by the growth of grain boundaries, see e.g. [2]. For further references and a more detailed discussion, we would like to refer the reader to the introduction in [9].

After the completion of this paper, Theorem 1.2 was extended by R. Mazzeo and M. Sáez in [10]. They provide a different existence proof and can handle also more than three half-lines.

The rest of the paper is organized as follows. We derive the equation for selfsimilarly expanding curves in Section 2. Existence and continuous dependence on the data for a self-similarly expanding curve starting at a fixed point at a fixed time which is asymptotic to a given half-line is shown in Section 3. We use this to construct a solution in Section 4 and prove uniqueness in Section 5. In Appendix A, we indicate how to find other self-similarly expanding solutions and mention some open problems. 
Acknowledgements. We want to thank Bernold Fiedler, Gerhard Huisken, Tom Ilmanen, Stefan Liebscher, Roger Moser, Mariel Sáez and Tatiana Toro for discussions.

\section{Derivation of the equation}

Assume that a curve $\alpha_{i}(\cdot, t)$ is locally represented as graph $u(\cdot, t)$ over the real line. Then graph $u(\cdot, t)$ flows by curve shortening flow, if

$$
\dot{u}=\sqrt{1+u^{\prime 2}}\left(\frac{u^{\prime}}{\sqrt{1+u^{\prime 2}}}\right)^{\prime}=\frac{u^{\prime \prime}}{1+u^{\prime 2}} .
$$

Our networks are homothetically expanding. We will rotate our coordinate system such that the start points of $\alpha_{i}(\cdot, t)$ lie on the set $\left\{(0, y) \in \mathbb{R}^{2}: y \geq 0\right\}$.

Assume first that a curve $\alpha_{i}(\cdot, t)$ is represented as $\operatorname{graph} u(\cdot, t), u(\cdot, t)$ : $\mathbb{R}_{\geq 0} \equiv\{x \in \mathbb{R}: x \geq 0\} \rightarrow \mathbb{R}$. For a homothetically expanding solution, the slope of $u$ at $(0, u(0, t)) \in \mathbb{R}^{2}$ is independent of $t$,

$$
\left.\frac{\partial}{\partial x} u(x, t)\right|_{x=0}=\text { const. }
$$

Our curves expand self-similarly if the graphs at different times differ by a homothety as follows

$$
\{(x, u(x, t)): x>0\}=\lambda(t) \cdot\{(y, u(y, 1 / 2)): y>0\}
$$

for some increasing function $\lambda(t): \mathbb{R}_{+} \rightarrow \mathbb{R}_{+}$. In view of the geometrically invariant formulation of mean curvature flow

$$
\frac{d}{d t} X=-H v
$$

where the embedding vector $X$ scales like $\lambda(t)$, the mean curvature $H$ like $\frac{1}{\lambda(t)}$, and the unit normal $v$ is scaling invariant, we deduce that $\dot{\lambda} \cdot \lambda=1$. As $\lambda(0)=0$, we have $\lambda(t)=\sqrt{2 t}$. So it suffices to describe an asymptotically expanding solution at some fixed time $t_{0}$. We may assume that $t_{0}=1 / 2$ and obtain $\dot{\lambda}\left(t_{0}\right)=\lambda\left(t_{0}\right)=1$. We substitute $y=y(x, t)=\frac{1}{\lambda(t)} x$ and differentiate (2.2) to get

$$
\begin{aligned}
\frac{\partial}{\partial t} u(x, t) & =\dot{\lambda} u(y, 1 / 2)-\frac{\dot{\lambda}}{\lambda} \frac{\partial}{\partial y} u(y, 1 / 2) x, \\
\frac{\partial}{\partial x} u(x, t) & =\frac{\partial}{\partial y} u(y, 1 / 2), \\
\frac{\partial^{2}}{\partial x^{2}} u(x, t) & =\frac{1}{\lambda(t)} \frac{\partial^{2}}{\partial y^{2}} u(y, 1 / 2) .
\end{aligned}
$$


We use these relations in (2.1) and obtain

$$
\dot{\lambda}(t)\left(u(y, 1 / 2)-\frac{x}{\lambda(t)} \frac{\partial}{\partial y} u(y, 1 / 2)\right)=\frac{1}{\lambda(t)}\left(\frac{\frac{\partial^{2}}{\partial y^{2}} u(y, 1 / 2)}{1+\left(\frac{\partial}{\partial y} u(y, 1 / 2)\right)^{2}}\right),
$$

where $x=\lambda(t) y$. At $t=1 / 2$, we get

$$
u-x u^{\prime}=\frac{u^{\prime \prime}}{1+\left(u^{\prime}\right)^{2}} .
$$

Note that at $t=1 / 2$, we have $x=y$ and $u^{\prime}=\frac{\partial}{\partial x} u=\frac{\partial}{\partial y} u$. So we study the initial value problem

$$
u-x u^{\prime}=\frac{u^{\prime \prime}}{1+\left(u^{\prime}\right)^{2}}, \quad x>0,
$$

for given $u(0)>0$ and $u^{\prime}(0)$. If $u$ is a solution to (2.3), then so is $-u$. Solutions with $u(0)=0$ are straight half-lines. Thus it suffices to study solutions with $u(0)>0$.

\section{Existence of one curve}

In this section, we show that for every point $P \in \mathbb{R}^{2}$ and every half-line $l_{1}$, there exists a self-similarly expanding curve as described before Theorem 1.2 that starts at $P$ and is asymptotic to $l_{1}$ at infinity. This curve $\alpha_{1}$ fulfills all the conditions mentioned before Theorem 1.2 that can be fulfilled by a single curve.

Lemma 3.1. As long as a solution to (2.3) exists, i.e. on a maximal interval $0 \leq$ $x<x_{\text {max }}, u$ is a strictly convex function.

Proof. Standard theory for ordinary differential equations implies that a unique smooth solution $u$ to (2.3) (with initial conditions for $u(0)>0$ and $u^{\prime}(0)$ ) exists on a maximal interval $0 \leq x<x_{\max } \leq \infty$. Our initial conditions with $u(0)>0$ and the differential equation ensure that $u^{\prime \prime}(0)>0$. Suppose that there exists $x_{0}>0$ such that $u^{\prime \prime}\left(x_{0}\right)=0$. We then have $u\left(x_{0}\right)-x_{0} u^{\prime}\left(x_{0}\right)=0$. The point $\left(x_{0}, u\left(x_{0}\right)\right)$ lies on some straight line through the origin. At $x_{0}$, the slopes of that straight line and of $u$ coincide. Note that this straight line solves our differential equation for all $x \in \mathbb{R}$ and is the only solution $w$ on a maximal existence interval of that equation with $w\left(x_{0}\right)=u\left(x_{0}\right)$ and $w^{\prime}\left(x_{0}\right)=u^{\prime}\left(x_{0}\right)$. As solutions to (2.3) (with given initial conditions) are unique, we see that graph $u$ is contained in a straight line, a contradiction.

Lemma 3.2. The initial value problem (2.3) has a solution for all $x \geq 0$. 
Proof. If $u^{\prime}(0)$ is negative, the convexity of $u$ implies that $\left|u^{\prime}\right|$ is decreasing. Thus $\left|u^{\prime}\right|$ is bounded as long as $u^{\prime}$ is negative. So, for bounded values of $x, u$ is bounded there. We deduce that $u$ exists for all $x \geq 0$ or $u^{\prime}$ becomes non-negative. We may assume the latter.

So we can find $\varepsilon>0$ in the maximal existence interval such that $u^{\prime}(\varepsilon) \geq 0$. The convexity of $u$ ensures that $u^{\prime}(x) \geq 0$ for $x>\varepsilon>0$. As $u$ is a strictly convex function, the differential equation implies that $u-x u^{\prime}>0$. So we deduce that

$$
u>x u^{\prime} \geq \varepsilon u^{\prime} \quad \text { for } x>\varepsilon>0 .
$$

Therefore, according to the maximum principle for ordinary differential equations, $u$ can grow at most exponentially for $x>\varepsilon$. Moreover, $u>\varepsilon u^{\prime}$ gives also a bound on $u^{\prime}$. Thus $u$ exists for all $x \geq 0$.

As solutions to (2.3) exist for all $x \geq 0$, we will assume in the following that any solution $u$ exists on an interval containing $[0, \infty)$. Note that if $u:[0, \infty) \rightarrow \mathbb{R}$ is a solution to (2.3), then $u(-x)$ is a solution to (2.3) with $u:(-\infty, 0] \rightarrow \mathbb{R}$. Thus it suffices to study properties of $u$ on $[0, \infty)$.

In order to prove that graph $u$ converges to a straight line through the origin for $x \rightarrow \infty$, it is useful to consider the quantity $u-x u^{\prime}$ which vanishes precisely when graph $u$ is contained in such a straight line.

Lemma 3.3. For a solution $u$ to (2.3), $u-x u^{\prime}$ is exponentially decaying in $x$.

Proof. As $u$ is strictly convex, the equation implies that $u-x u^{\prime}>0$. We use (2.3) to derive an evolution equation for $u-x u^{\prime}$ on $\{x \geq \varepsilon>0\}$

$$
\left(u-x u^{\prime}\right)^{\prime}=-x u^{\prime \prime}=-x\left(u-x u^{\prime}\right)\left(1+\left(u^{\prime}\right)^{2}\right) \leq-\varepsilon\left(u-x u^{\prime}\right) .
$$

Therefore $u-x u^{\prime}$ is exponentially decreasing in $x$.

Near $x=0$, the graph of a solution $u$ intersects lines through the origin of the form $\{(x, a x): x \in \mathbb{R}\}, a \gg 1$. By convexity, $u^{\prime}$ is bounded below, so $u$ will never intersect lines of the form $\{(x, a x): x \in \mathbb{R}\}$ for $a \ll-1$. In order to find the line where graph $u$ becomes asymptotic to, we show in the next corollary that graph $u$ intersects each line through the origin at most once. We will then prove that for large values of $x$, graph $u$ is asymptotically close to the line through the origin with slope equal to the infimum of slopes of lines through the origin which intersect graph $u$.

Lemma 3.4. The function $x \mapsto \frac{u(x)}{x}$ is strictly decreasing.

Proof. We have

$$
\left(\frac{u(x)}{x}\right)^{\prime}=\frac{u^{\prime}}{x}-\frac{u}{x^{2}}=\frac{x u^{\prime}-u}{x^{2}}<0
$$

The lemma follows. 
This implies in particular:

Corollary 3.5. Let $a \in \mathbb{R}$ and let $u$ be a solution to $(2.3)$ with $u(0)>0$. If $u\left(x_{0}\right)=$ ax for some $x_{0}>0$, then $u(x)>$ ax for $0 \leq x<x_{0}$ and $u(x)<$ ax for $x>x_{0}$. Thus the graphs of $u$ and $x \mapsto$ ax intersect at most once for $x \geq 0$.

The following lemma implies for some $a>0$ that graph $\left.u\right|_{\mathbb{R}_{\geq 0}}$ is asymptotic to a half-line of the form graph $\left(\mathbb{R}_{\geq 0} \ni x \mapsto a x\right)$ at infinity.

Lemma 3.6. There exists $a \in \mathbb{R}$ such that $u(x)-a x \rightarrow 0$ exponentially in $C^{\infty}$ for $x \rightarrow \infty$.

Proof. We define $a:=\lim _{x \rightarrow \infty} \frac{u(x)}{x}$. As $u$ is convex, $u$ decays at most linearly. Thus Lemma 3.4 implies that $a \in \mathbb{R}$ is well-defined. According to Lemma 3.3, $u(x)-x u^{\prime}(x)$ converges exponentially to zero for $x \rightarrow \infty$. Therefore we have $\lim _{x \rightarrow \infty} u^{\prime}(x)=\lim _{x \rightarrow \infty} \frac{u(x)}{x}=a$. In view of Equation (2.3) we obtain that $u^{\prime \prime}(x) \rightarrow 0$ exponentially as $x \rightarrow \infty$. Integrating yields that also $u^{\prime}(x) \rightarrow a$ exponentially for $x \rightarrow \infty$. Using Lemma 3.3 once again, we get that $u(x)-a x \rightarrow 0$ exponentially in $C^{0}$ as $x \rightarrow \infty$. Differentiating (2.3) yields bounds on higher derivatives of $u$ and interpolation implies exponential convergence.

Solutions to (2.3) fulfill useful monotonicity properties. We will derive these in the following lemmata.

Lemma 3.7. Let $u$ and $v$ be solutions to $(2.3)$ with $u(0)>v(0)>0$ and $u^{\prime}(0)=$ $v^{\prime}(0)$. Then $u-v$ is positive everywhere. This difference is even strictly increasing in $x$ for $x \geq 0$.

Proof. The differential equation implies that $u^{\prime \prime}(0)>v^{\prime \prime}(0)$. Thus the difference $u-v$ is strictly increasing in $x$ for small $x>0$. Consider a maximal interval $\left(0, x_{0}\right), 0<x_{0} \leq \infty$, where $u^{\prime}-v^{\prime}>0$. We want to exclude that $x_{0}<\infty$. If this is the case, we get $u^{\prime}\left(x_{0}\right)-v^{\prime}\left(x_{0}\right)=0$ and $u^{\prime \prime}\left(x_{0}\right)-v^{\prime \prime}\left(x_{0}\right) \leq 0$. As $u-v$ is strictly increasing on $\left(0, x_{0}\right)$, we have $u\left(x_{0}\right)>v\left(x_{0}\right)$. We obtain

$$
\begin{aligned}
0 & \geq u^{\prime \prime}\left(x_{0}\right)-v^{\prime \prime}\left(x_{0}\right) \\
& =\left(1+\left(u^{\prime}\left(x_{0}\right)\right)^{2}\right)\left(u\left(x_{0}\right)-x_{0} u^{\prime}\left(x_{0}\right)\right)-\left(1+\left(v^{\prime}\left(x_{0}\right)\right)^{2}\right)\left(v\left(x_{0}\right)-x_{0} v^{\prime}\left(x_{0}\right)\right) \\
& =\left(1+\left(u^{\prime}\left(x_{0}\right)\right)^{2}\right)\left(u\left(x_{0}\right)-v\left(x_{0}\right)\right)>0,
\end{aligned}
$$

a contradiction. Thus $x_{0}=\infty$ and $u-v$ is strictly increasing in $x$.

Lemma 3.8. Let $u$ and $v$ be solutions to $(2.3)$ with $u(0)=v(0)>0$ and $u^{\prime}(0)>$ $v^{\prime}(0)$. Then $u-v$ is positive for $x>0$. This difference is even strictly increasing in $x$ for $x \geq 0$. 
Proof. By assumption, $u-v$ is strictly increasing for small $x \geq 0$. Suppose that there is a smallest $x_{0}>0$ such that $u^{\prime}\left(x_{0}\right)-v^{\prime}\left(x_{0}\right)=0$. At $x_{0}$, we have $u\left(x_{0}\right)>$ $v\left(x_{0}\right)$ and $u^{\prime \prime}\left(x_{0}\right) \leq v^{\prime \prime}\left(x_{0}\right)$. We deduce there

$$
u-x u^{\prime}=\frac{u^{\prime \prime}}{1+\left(u^{\prime}\right)^{2}} \leq \frac{v^{\prime \prime}}{1+\left(v^{\prime}\right)^{2}}=v-x v^{\prime},
$$

so $u\left(x_{0}\right) \leq v\left(x_{0}\right)$, a contradiction.

Not only for the functions, but also for the asymptotic slopes, we obtain a strict monotonicity.

Lemma 3.9. Let $u$ and $v$ be solutions to (2.3) with $u(0) \geq v(0)>0$ and $u^{\prime}(0) \geq$ $v^{\prime}(0)$, but $\left(u(0), u^{\prime}(0)\right) \neq\left(v(0), v^{\prime}(0)\right)$. Then

$$
\lim _{x \rightarrow \infty} \frac{u(x)}{x}>\lim _{x \rightarrow \infty} \frac{v(x)}{x} .
$$

Proof. Use Lemmata 3.7 and 3.8 to see that $u(x)-v(x)$ is positive and increasing in $x$ for $x>0$. As the exponential convergence in Lemma 3.6 is uniform for initial values $\left(u(0), u^{\prime}(0)\right)$ in a compact set, the limits of $\frac{u(x)}{x}$ and $\frac{v(x)}{x}$ differ.

For solutions $u$ to (2.3) with $u^{\prime}(0)=0$, we want to study the slopes $u^{\prime}$ near infinity. For $u(0)=0$, we obtain that $u(x)=0$ is a solution. In the following lemma, we prove that the slopes near infinity become large for large values of $u(0)$.

Lemma 3.10. For given $a>0$, there exists $h \gg 1$, such that a solution $u$ to (2.3) with $u^{\prime}(0)=0$ and $u(0) \geq h$ has slope bigger than a near infinity,

$$
\lim _{x \rightarrow \infty} u^{\prime}(x)>a .
$$

According to Lemma 3.4 and the proof of Lemma 3.6, we also obtain that $\lim _{x \rightarrow \infty} \frac{u(x)}{x}>a$ and $u(x)>a x$ for $x \geq 0$.

Proof. If $u^{\prime}\left(\frac{1}{2}\right) \geq h$ for some $h \gg 1$, the lemma is proven. Details for this argument can be found at the end of this proof. Otherwise we have $0 \leq u^{\prime}(x) \leq h$ and $h \leq u(x)$ for all $0 \leq x \leq \frac{1}{2}$ as $u$ is convex. We estimate there

$$
u^{\prime \prime}=\left(1+\left(u^{\prime}\right)^{2}\right) \cdot\left(u-x u^{\prime}\right) \geq 1 \cdot\left(h-\frac{1}{2} h\right)=\frac{1}{2} h .
$$

Thus

$$
u^{\prime}\left(\frac{1}{2}\right) \geq \int_{0}^{1 / 2} u^{\prime \prime}(x) d x \geq \frac{1}{4} h
$$

By convexity and Lemma 3.6, $\lim _{x \rightarrow \infty} u^{\prime}(x)$ exists and $\lim _{x \rightarrow \infty} u^{\prime}(x)>u^{\prime}\left(\frac{1}{2}\right) \geq \frac{1}{4} h$. 
We will later use the following variant of Lemma 3.10.

Lemma 3.11. Let $a>0, \mu>0$. Then there exists $h \gg 1$ such that every solution $u$ to (2.3) with $u(0) \geq h$ and $\lim _{x \rightarrow \infty} u^{\prime}(x) \leq$ a fulfills $u^{\prime}(0) \leq-\mu$.

Proof. As the asymptotic slope is monotone in the height and in the initial slope, see Lemma 3.9, it suffices to show that a solution to (2.3) with $u(0)=h$ and $u^{\prime}(0)=-\mu$ has an asymptotic slope bigger than $a$ at infinity, if $h \gg 1$ is chosen sufficiently large.

According to Lemma 3.9, we may assume that $\mu$ is big; we assume that $\mu>a$. As long as $u \geq \frac{h}{2}$ on the interval $0 \leq x \leq 1$, we estimate

$$
u^{\prime \prime}(x)=\left(1+\left(u^{\prime}(x)\right)^{2}\right)\left(u(x)-x u^{\prime}(x)\right) \geq u(x)-x u^{\prime}(x) \geq \frac{h}{2}-\left|u^{\prime}(x)\right| .
$$

If $u^{\prime}(x)>\mu$ for some $x>0$, convexity implies that the lemma holds. Otherwise, we may assume that $\frac{h}{4} \geq \mu$ and use convexity to estimate further

$$
u^{\prime \prime}(x) \geq \frac{h}{2}-\mu \geq \frac{h}{4} .
$$

A solution to $v^{\prime \prime}(x)=\frac{h}{4}$ with initial conditions $v(0)=h, v^{\prime}(0)=-\mu$ is given by $v(x)=h-\mu x+\frac{h}{8} x^{2}$. We have $u(x) \geq v(x)$, for $0 \leq x \leq 1$ provided that $u(x) \geq \frac{h}{2}$. The minimum of $v$ is attained at $x=\frac{4 \mu}{h}$ and equals $h-\frac{2 \mu^{2}}{h}$ which is bigger than $\frac{h}{2}$ if $h>2 \mu$. We will assume this in the following. This justifies the assumption that $u \geq \frac{h}{2}$ on the interval $0 \leq x \leq 1$ for large values of $h$. We obtain that $u(x) \geq v(x)$ for $0 \leq x \leq 1$. We deduce especially that $u(1) \geq v(1) \geq \frac{9}{8} h-\mu$. Assume that $h \geq 8 \mu$. Then $u(1)-u(0) \geq 8 \mu$. Therefore, we find $x \in[0,1]$ with $u^{\prime}(x) \geq 8 \mu$. Now the lemma follows as $u$ is convex.

In the following lemma, we show that for a prescribed half-line $\{(x, a x): x \in \mathbb{R}\}$, $a>0$, there exists an initial height $h$ such that the corresponding solution to (2.3) converges to that half-line near infinity.

Lemma 3.12. Let $a>0$. Then there exists an initial height $u(0)=h$ such that the corresponding solution to (2.3) with $u^{\prime}(0)=0$ fulfills

$$
\lim _{x \rightarrow \infty} u(x)-a x=0 .
$$

Proof. Apply graphical mean curvature flow to the initial value $u_{0}(x)=a \cdot|x|$ and use the results of $[3,4,11]$. If $u: \mathbb{R} \times[0, \infty)$ is such a solution, then $u(\cdot, 1 / 2)$ is as claimed in the lemma.

We can also prove that $a$ and $h$ depend continuously on each other. 
Lemma 3.13. Let $a$ and $h$ be related via solutions $u$ to (2.3) as in Lemma 3.12. By reflection of graph $u$, we extend this to a map $\mathbb{R} \ni h \mapsto a \in \mathbb{R}$. Then this map is a homeomorphism with $a(h) \rightarrow \pm \infty$ for $h \rightarrow \pm \infty$.

Proof. Lemma 3.10 ensures that $a(h) \rightarrow \pm \infty$ for $h \rightarrow \pm \infty$. Surjectivity follows from Lemma 3.12. Lemma 3.9 implies the injectivity. Continuity of the map follows from the continuous dependence on initial data of solutions to ordinary differential equations, applied to $x$ in compact intervals, and the uniform exponential convergence, Lemma 3.6. Now standard topology, see e.g. [12, Satz 8.12 and Satz 8.24], implies that a bijective, continuous and proper map from $\mathbb{R}^{n} \rightarrow \mathbb{R}^{n}$ is in fact a homeomorphism. Thus $h \mapsto a(h)$ is a homeomorphism.

We assume now that we are given a half-line $l$ (starting at the origin) in $\mathbb{R}^{2}$ and a point $P$. Our aim is to find a self-similarly expanding solution to curve shortening flow with start point at $t=1 / 2$ equal to $P$ which is asymptotic to the given half-line $l$. It suffices to consider the elliptic problem derived in Section 2. We may rotate the situation such that $P$ lies in the set $\{(0, y): y \geq 0\}$. If $P$ lies on the half-line $l$, the claim is obvious. Assume thus that $P \neq 0$ and that the half-line is given as $\{(x, a x): x \geq 0\}, a \in \mathbb{R}$.

The idea to find the corresponding curve is as follows. For $h \in \mathbb{R}$, we consider solutions to (2.3) with $u(0)=h$ and $u^{\prime}(0)=0$ for all $x \in \mathbb{R}$. We rotate these solutions around the origin such that the half-line graph $\left(\mathbb{R}_{\geq 0} \ni x \mapsto a x\right)$ with $a$ as above is mapped to the given half-line $l$. The resulting curves for different values of $h$ are visualized in Figure 1.

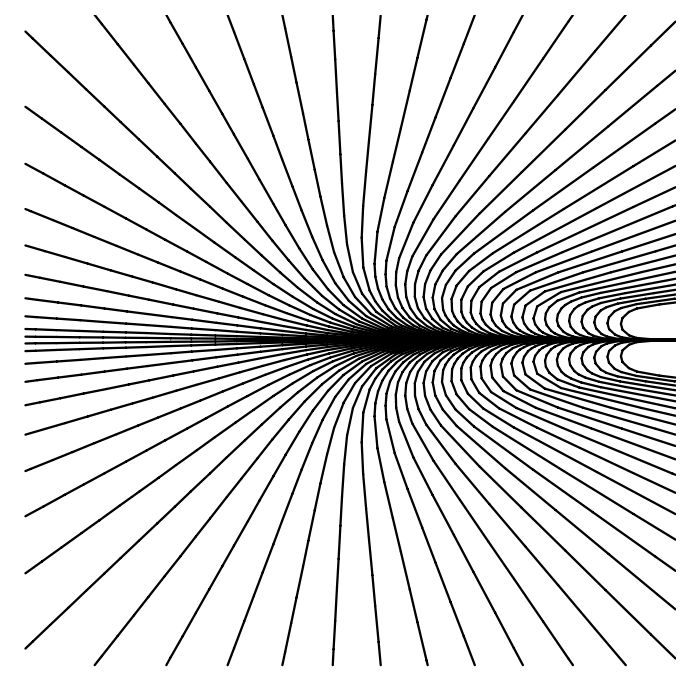

Figure 1.

In the picture, these curves foliate $\mathbb{R}^{2}$ nicely. So it suffices to pick the curve that passes through $P$. Note also that for different values of $P$, the corresponding tangent vectors to the respective curve seem to vary continuously. 
The facts visualized here, are proved as follows. Lemma 3.13 implies that the rotations depend continuously on $h$, so we get a family of curves parameterized by $h \in \mathbb{R}$, that depend continuously (measured in $C_{l o c}^{0}$ for appropriate parameterizations) on $h$. For $|h|$ sufficiently large, due to Lemma 3.10 and the convexity, the rotated curves are contained in a cone of small opening angle around the half-line $l$. We may assume that $P$ has a neighborhood disjoint to that cone. When we vary $h$, one of the half-lines to which a curve is asymptotic to at infinity always coincides with the half-line $l$ while the other turns by $360^{\circ}$. This is only possible if there exists a curve in the family considered, that contains $P$ and is asymptotic to $l$. Applying Lemma 3.8 and the exponential convergence, Lemma 3.6, similarly to the proof of Lemma 3.13, we see that the curve is also unique. Applying again [12, Satz 8.12 and Satz 8.24] to a map induced from the family of curves considered, we see that $(x, h) \in \mathbb{R}^{2}$ such that $(x, u(x))$, where $u$ solves $(2.3)$ with $u(0)=h$ and $u^{\prime}(0)=0$, is rotated to $P$, depends continuously on $P$. This implies in particular that the tangent vector to that curve at $P$ depends continuously on $P$. By direct inspection, we see that such is also true if $P$ lies on $l$. We have thus proved

Lemma 3.14. Let $P \in \mathbb{R}^{2}$ and let $l$ be a half-line starting at the origin. Then there exists a solution $u$ to $(2.3)$ and a rotation $R$ about the origin such that $P \in$ $R$ (graph $u$ ) and $R\left(\right.$ graph $\left.\left.u\right|_{\mathbb{R}_{\geq 0}}\right)$ is asymptotic to $l$ at infinity. Assume that $P=$ $R\left(x_{0}, u\left(x_{0}\right)\right)$. Then the tangent vector $R\left(\frac{\left(1, u^{\prime}\right)}{\sqrt{1+\left(u^{\prime}\right)^{2}}}\left(x_{0}\right)\right)$ depends continuously on $P$.

It also follows that the tangent vector varies continuously if we rotate the fixed half-line. We do not use this observation in the present paper.

\section{Existence of three curves}

In this section, we show that there exists at least one start point $P \in \mathbb{R}^{2}$ for which the problem described in Section 1 can be solved.

We have seen in Section 3, that for any $P \in \mathbb{R}^{2}$, there exist three curves that fulfill the conditions in Section 1, but do not necessarily meet at the start point at $120^{\circ}$ angles. We also have to show that these curves only have their start point in common.

The following lemma concerns embeddedness of the curves.

Lemma 4.1. Different self-similarly expanding curves which have a common start point do not intersect outside this start point.

Proof. We may rotate our coordinate system such that the start point lies on the half-line $\{(0, y): y \geq 0\}$. These curves are always graphs over $\{x \geq 0\}$ or over $\{x \leq 0\}$ or are part of the axis $\{x=0\}$. Thus it suffices to study two curves that are represented as graphs over the half-line $\{x \geq 0\}$. They start at the same height and have different initial slopes as they are not identical. Thus Lemma 3.8 implies that these two curves meet only for $x=0$. 
In order to show that there exists at least one start point $P$, where the three curves meet at an angle of $120^{\circ}$, we fix the half-lines and vary $P$. For each $P$, we sum up the tangential directions $\left(T_{i}\right)_{i}$ at $P$. This gives a continuous vector field $V=$ $\sum_{i=1}^{3} T_{i}$ on $\mathbb{R}^{2}$. We will show that for points $P$ far from the origin, we always get $\langle P, V(P)\rangle<0$. We aim at applying the Brouwer fixed point theorem to show that there exists a $P_{0}$ such that $V\left(P_{0}\right)=0$. For this start point, the $120^{\circ}$ condition is fulfilled.

The proof of Theorem 1.2 is thus finished provided that we can show that $\langle P, V(P)\rangle<0$ for all $P \in \mathbb{R}^{2}$ such that $|P|$ is sufficiently large. This follows from Lemma 3.11. It states that for $|P|$ sufficiently large, a self-similar solution has to start almost in the direction towards the origin in order to be asymptotic to a half-line that encloses an angle estimated from below (by a positive constant) to the half-line starting at the origin and passing through $P$. Thus the corresponding tangent vector at $P$ almost equals $-\frac{P}{|P|}$. Thus, having fixed three distinct halflines starting at the origin, the half-line starting at the origin and passing through $P$ encloses an angle estimated from below to at least two of them. Thus at least two of the three vectors $T_{i}$ at $P$ are almost equal to $-\frac{P}{|P|}$. This suffices to ensure that $\langle P, V(P)\rangle=\sum_{i=1}^{3}\left\langle P, T_{i}\right\rangle<0$ on $\partial B_{R}(0)$ for $R \geq 1$ sufficiently large. We may even assume that $P+V(P)$ lies inside $B_{R}(0)$ for all $P \in B_{R}(0)$. The map $P \mapsto P+V(P): \bar{B}_{R}(0) \rightarrow \bar{B}_{R}(0)$ is continuous. Hence Brouwer's fixed point theorem implies that it has a fixed point $P_{0}$. This is equivalent to $V\left(P_{0}\right)=0$. Therefore, we find some $P_{0} \in \mathbb{R}^{2}$ such that we have $\sum_{i=1}^{3} T_{i}=0$ there, i.e. every two tangent vectors at $P_{0}$ enclose an angle of $120^{\circ}$. This completes the existence part of the proof of Theorem 1.2.

\section{Uniqueness}

To prove uniqueness of a homothetically expanding network for three given distinct half-lines, we will first show that the angle, at which self-similarly expanding curves, asymptotic to a fixed half-line, intersect another self-similarly expanding curve, is monotone.

Let $l$ be a given-half line in $\mathbb{R}^{2}$, which we can assume to coincide with the set $\left\{(x, 0) \in \mathbb{R}^{2} \mid x \geq 0\right\}$. Furthermore let $c_{h}: \mathbb{R} \rightarrow \mathbb{R}^{2}$ be the self-similarly expanding curves asymptotic to this half-line, as considered in Section 3, parameterized such that

$$
\operatorname{dist}\left(c_{h}(t), l\right) \rightarrow 0 \quad \text { as } t \rightarrow \infty
$$

We distinguish these curves by the parameter $h$, where $h$ is such that

$$
\operatorname{dist}\left(\operatorname{Im}\left(c_{h}\right),(0,0)\right)=|h|,
$$

and we take $h$ to be positive if $c_{h}$ lies in the upper half-plane and negative, if $c_{h}$ lies in the lower half-plane. 
Let $b: \mathbb{R} \rightarrow \mathbb{R}^{2}$ be a further self-similarly expanding curve, asymptotic to a different half line in $\mathbb{R}^{2}$, i.e. there exists an $h \in \mathbb{R}$ and a rotation $R \neq$ id such that $b=R \circ c_{h}$. Note that by Lemma 3.8, a curve $c_{h}$ intersects $b$ at most once. Assume now that we have two curves $c_{h_{1}}, c_{h_{2}}$ which intersect $b$ in points $p_{1}$ and $p_{2}$, respectively. We assume further that $h_{1}, h_{2}$ are such that $p_{1}$ lies before $p_{2}$ with respect to the parameterization of $b$. Let $\alpha_{i}$ be the angle between the tangent vector $T_{i}$ to $c_{h_{i}}$ and the tangent vector $T_{b}$ at $p_{i}$, where $i=1,2$.

Lemma 5.1. We have

$$
\alpha_{2}-\alpha_{1}=2|A|,
$$

where $A$ is the domain enclosed by these three curves as indicated in the picture and $|A|$ denotes its area.

Note that by the exponential convergence of $c_{h_{1}}(t)$ and $c_{h_{2}}(t)$, parameterized as graphs over the $x$-axis, to $l$ as $t \rightarrow \infty$, the area $|A|$ is finite.

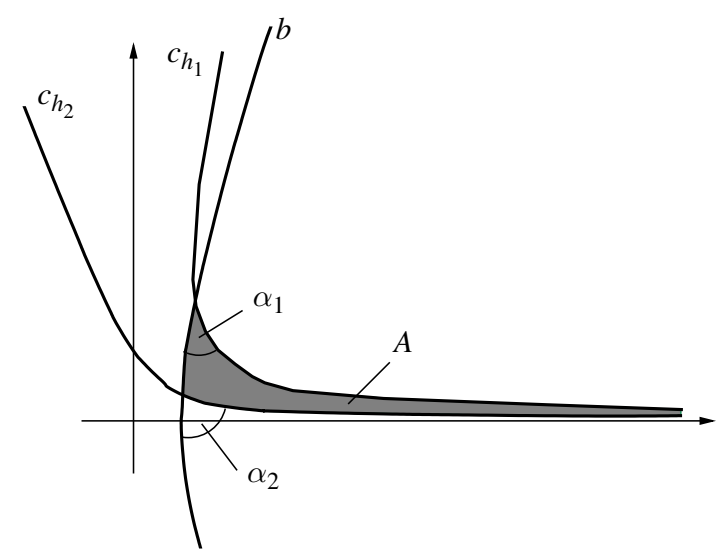

Proof. By an analogous derivation as in Section 2 it is easy to see that equation (2.1) for a self-similarly expanding curve can be written in the coordinate free form

$$
H=-\langle X, v\rangle,
$$

where the sign of $H$ is chosen such that $-H v$ is the (mean) curvature vector, $v$ is a choice of unit normal and $X$ is the position vector field $\left(x_{1}, x_{2}\right) \mapsto\left(x_{1}, x_{2}\right)$. By the divergence theorem we can compute

$$
\begin{aligned}
2|A| & =\int_{A} \operatorname{div}(X) d x=\int_{\partial A}\langle X, \nu\rangle d \sigma=-\int_{\partial A} \kappa d \sigma \\
& =-\left(2 \pi-\pi-\left(\pi-\alpha_{1}\right)-\alpha_{2}\right)=\alpha_{2}-\alpha_{1},
\end{aligned}
$$

where $v$ is the outward unit normal to $\partial A$ and we have oriented $\partial A$ counterclockwise. 


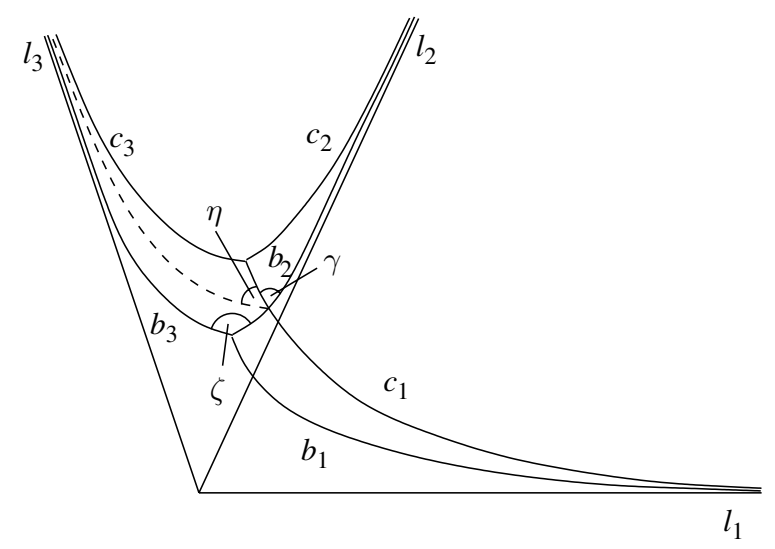

Figure 2.

We can use this monotonicity to prove uniqueness of a homothetically expanding network. Let $l_{1}, l_{2}, l_{3}$ be three given distinct half-lines, meeting at the origin and assume that there exist two different homothetically expanding networks, satisfying the balancing condition at the triple point such that both are asymptotic to the three half-lines. Let the first network consist of the three curves $b_{1}, b_{2}, b_{3}$, meeting at the triple point $p$ and the second consist of $c_{1}, c_{2}, c_{3}$, meeting at the triple point $q$. To avoid notational confusion, let us assume that we have a configuration as in Figure 2. It is easily seen that this argument works for any such configuration. In the case of a degenerate configuration, i.e. for example if $\operatorname{Im}\left(b_{1}\right) \subset \operatorname{Im}\left(c_{1}\right)$, it follows easily from Lemma 5.1 that we get a contradiction along $c_{1}$. In the picture, we have introduced the additional self-similarly expanding curve, asymptotic to $l_{3}$ (dotted in the picture), starting at the intersection point $r$ of $b_{2}$ and $c_{1}$. This curve intersects the curve $c_{1}$ at an angle $\eta$ as shown above. The angle between $b_{2}$ and $c_{1}$ at $r$ is denoted by $\gamma$. Note that by Lemma 5.1, applied along $c_{1}$, between $q$ and $r$, we have that $\eta<\pi / 3$, and $\gamma<\pi / 3$. Thus $\eta+\gamma<2 \pi / 3$. Applying the monotonicity now along $b_{2}$, between $r$ and $p$, we see that also $\zeta<2 \pi / 3$ which yields a contradiction. This completes the proof of Theorem 1.2.

\section{A. Further results and open problems}

In the following remark, we use the term network to describe a properly embedded graph with finitely many vertices and (possibly unbounded) edges, where at vertices three edges meet at $120^{\circ}$ angles.

Remark A.1. There is no homothetically expanding network $\left(M_{t}\right)_{t}$ which is asymptotic to at most six half-lines at infinity and non-trivial fundamental group.

Sketch of proof. Denote by $\Omega_{t}$ a connected component in the closure of the bounded components of $\mathbb{R}^{2} \backslash M_{t}$. The set $\Omega_{t}$ has not more corners, i.e. corners, where a curve leaves $\Omega_{t}$, than half-lines to which $M_{t}$ is asymptotic to at infinity. Note that $\Omega_{t} \neq \varnothing$ 
if and only if $M_{t}$ has non-trivial fundamental group. We wish to prove that $\Omega_{t}=\emptyset$. Let us assume the contrary. As $M_{t}$ is homothetically expanding, the volume of $\Omega_{t}$ is growing in $t$, hence

$$
\int_{\partial \Omega_{t}} \kappa<0 .
$$

On the other hand, the boundary of $\Omega_{t}$ is a closed loop, hence

$$
2 \pi=\int_{\partial \Omega_{t}} \kappa+c_{\text {out }} \frac{\pi}{3}-c_{\text {in }} \frac{\pi}{3},
$$

where $c_{\text {out }}$ denotes the corners on $\partial \Omega_{t}$, where the edge that is not part of $\partial \Omega_{t}$ leaves $\Omega_{t}$ and $c_{\text {in }}$ denotes the other corners along $\partial \Omega_{t}$. By assumption, we have $c_{\text {out }} \leq 6$. Clearly $c_{\text {in }} \geq 0$. We use these bounds and (A.1) to estimate the right-hand side of (A.2) and obtain a contradiction unless $\Omega_{t}=\emptyset$. Hence $M_{t}$ contains no closed loops.

Assume that we have again three distinct half-lines $l_{1}, l_{2}, l_{3}$, meeting at the origin, and let $P\left(l_{1}, l_{2}, l_{3}\right)$ be the triple point of the unique homothetically expanding network which is asymptotic to the three half-lines as in Theorem 1.2. We want to argue that $P\left(l_{1}, l_{2}, l_{3}\right)$ depends continuously on $l_{1}, l_{2}, l_{3}$. Note that the map $\Phi$ which maps the triple point $P$ and the angle $\vartheta$ of the three tangent directions in space at $P$ to the three asymptotic half-lines is continuous in $P$ and $\vartheta$ by the continuous dependence on initial data and the exponential convergence of the self-similarly expanding curves to half-lines. Theorem 1.2 implies that this map is one-to-one and onto. By Lemma 3.10, for $|P|$ sufficiently large, at least two of the half-lines are close to each other. Thus $\Phi$ is proper. So we can again apply [12, Satz 8.12 and Satz 8.24] to see that $\Phi$ is a homeomorphism. We obtain:

Lemma A.2. The triple point $P$ of a homothetically expanding network depends continuously on the three distinct asymptotic half-lines $l_{1}, l_{2}, l_{3}$.

Note that by uniqueness the triple point $P$ can lie on one of the half-lines, say $l_{2}$, only if the configuration is symmetric, i.e. the angle between $l_{1}$ and $l_{2}$ equals the angle between $l_{2}$ and $l_{3}$ and both are less or equal than the angle between $l_{1}$ and $l_{3}$. Furthermore, if the angles between $l_{1}$ and $l_{2}$ and between $l_{2}$ and $l_{3}$ are again equal, but bigger than the angle between $l_{1}$ and $l_{3}$, then the triple point is strictly contained in the smallest segment bounded by $l_{1}$ and $l_{3}$, see Lemma 3.9. More precisely, it lies on the continuation of $l_{2}$ into the segment bounded by $l_{1}$ and $l_{3}$.

Now assume that we have a non-symmetric configuration $l_{1}, l_{2}, l_{3}$, and the smallest segment is bounded by $l_{1}$ and $l_{3}$. We want to show that the triple point has to lie in this smallest segment, see Figure 3 . To see this we first rotate $l_{2}$ into the symmetric configuration, such that the segment bounded by $l_{1}$ and $l_{3}$ remains the smallest segment. Here we know that the triple point lies strictly inside the segment bounded by $l_{1}$ and $l_{3}$. Then we rotate $l_{2}$ back into its original position. Note that 


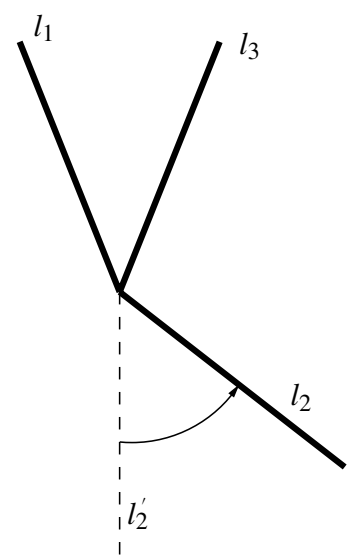

\section{Figure 3.}

while doing this we do not pass a symmetric configuration. Thus by Lemma A.2 the triple point remains in the smallest segment, which gives:

Lemma A.3. Let $l_{1}, l_{2}, l_{3}$ be three distinct half-lines meeting at the origin, such that one of the segments bounded by the half-lines is the unique smallest one. Then $P\left(l_{1}, l_{2}, l_{3}\right)$ lies in this smallest segment.

Remark A.4. A homothetically expanding network as above partitions $\mathbb{R}^{2}$ into three unbounded segments. Note that locally the change of enclosed volume under mean curvature flow is given by the integral over the (mean) curvature along the curve. In this non-compact setting, taking for example the network at $t=0$ as a reference, the change of renormalized area of each of the sectors is given by the asymptotic angle of the sector minus $2 \pi / 3$.

Remark A.5. T. Ilmanen [8] has studied the existence of smooth solutions which approximate two intersecting lines for small times and expand homothetically under curve shortening flow. In general, these solutions are not unique. As indicated in the picture,

\section{Figure 4.}

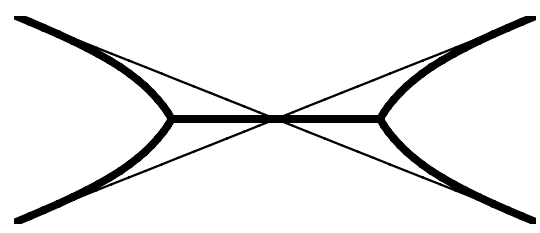

there exists another solution built from two networks as considered in this paper. The connecting segment is straight. Note that the triple points will be in sectors with an opening angle of less than $120^{\circ}$. Such a construction can be generalized, see also [1]. 
Open Problems A.6.

(i) Is there a simple (algebraic) relation involving the angles between the half-lines and

- the direction, in which the triple point moves?

- the tangent directions at the triple point?

- the distance between the origin and the triple point at $t=1 / 2$ ?

(ii) Show that there exists a solution to this initial value problem if the network consists initially of three (not necessarily straight) curves with common start points.

(iii) Show that in this situation, the forward blow-up around the triple point is a solution as constructed in this paper.

\section{References}

[1] K. A. BRAKKe, "The Motion of a Surface by its Mean Curvature", Mathematical Notes, Vol. 20, Princeton University Press, Princeton, N.J., 1978.

[2] L. BRONSARD and F. REITICH, On three-phase boundary motion and the singular limit of a vector-valued Ginzburg-Landau equation, Arch. Ration. Mech. Anal. 124 (1993), 355-379.

[3] K. ECKER and G. HUISKEn, Mean curvature evolution of entire graphs, Ann. of Math. 130 (1989), 453-471.

[4] K. ECKER and G. HUISKEN, Interior estimates for hypersurfaces moving by mean curvature, Invent. Math. 105 (1991), 547-569.

[5] M. E. GAGE and R. S. HAMiLton, The heat equation shrinking convex plane curves, J. Differential Geom. 23 (1986), 69-96.

[6] M. A. GRAYSOn, The heat equation shrinks embedded plane curves to points, J. Differential Geom. 26 (1987), 285-314.

[7] G. HUISKEN, A distance comparison principle for evolving curves, Asian J. Math. 2 (1998), $127-133$.

[8] T.ILmanen, "Lectures on Mean Curvature Flow and Related Equations", 1998, available from http: / /www. math. ethz.ch/ ilmanen/

[9] C. Mantegazza, M. Novaga and V. M. Tortorelli, Motion by curvature of planar networks, Ann. Scuola Norm. Sup. Pisa Cl. Sci. (5) 3 (2004), 235-324.

[10] R. MAZZEO and M. SÁEZ, Self similar expanding solutions of the planar network flow, arXiv:0704.3113v1 [math.DG].

[11] N. Stavrou, Selfsimilar solutions to the mean curvature flow, J. Reine Angew. Math. 499 (1998), 189-198.

[12] B. VON QuerenburG, "Mengentheoretische Topologie", Springer-Verlag, Berlin, 1973, Hochschultext.

Freie Universität Berlin

Arnimallee 6

14195 Berlin, Germany

Oliver.Schnuerer@math.fu-berlin.de

Felix.Schulze@math.fu-berlin.de 